\title{
A NOTE ON $L_{2}$-ESTIMATES FOR STABLE INTEGRALS WITH DRIFT
}

\author{
VLADIMIR KURENOK
}

Abstract. Let $X$ be of the form $X_{t}=\int_{0}^{t} b_{s} d Z_{s}+\int_{0}^{t} a_{s} d s, t \geq 0$, where $Z$ is a symmetric stable process of index $\alpha \in(1,2)$ with $Z_{0}=0$. We obtain various $L_{2}$-estimates for the process $X$. In particular, for $m \in \mathbb{N}, t \geq 0$, and any measurable, nonnegative function $f$ we derive the inequality

$$
\mathbf{E} \int_{0}^{t \wedge \tau_{m}(X)}\left|b_{s}\right|^{\alpha} f\left(X_{s}\right) d s \leq N\|f\|_{2, m} .
$$

As an application of the obtained estimates, we prove the existence of solutions for the stochastic equation $d X_{t}=b\left(X_{t-}\right) d Z_{t}+a\left(X_{t}\right) d t$ for any initial value $x_{0} \in \mathbb{R}$.

\section{INTRODUCTION}

Let $f: \mathbb{R} \rightarrow[0, \infty)$ be a measurable function and $X$ be a stochastic process of the form

$$
X_{t}=x_{0}+\int_{0}^{t} b_{s} d Z_{s}+\int_{0}^{t} a_{s} d s, \quad x_{0} \in \mathbb{R}, \quad t \geq 0,
$$

where $Z$ is a one-dimensional symmetric stable process of index $\alpha \in(0,2]$ with $Z_{0}=0$, and $\left(b_{s}\right)$ and $\left(a_{s}\right)$ are two processes such that the corresponding stochastic and Lebesgue integrals are well-defined.

In this note we shall prove some global and local $L_{2}$-estimates for processes of the form (1.1) when $\left|a_{s}\right| \leq K\left|b_{s}\right|^{\alpha}$ for a constant $K>0$. In particular, let $m \in \mathbb{N}$ and $\tau_{m}(X):=\inf \left\{t \geq 0:\left|X_{t}\right| \geq m\right\}$. Then, for all $t \geq 0$, it holds that

$$
\mathbf{E} \int_{0}^{t \wedge \tau_{m}(X)}\left|b_{s}\right|^{\alpha} f\left(X_{s}\right) d s \leq N\|f\|_{2, m},
$$

where $\|f\|_{2, m}:=\left(\int_{-m}^{m} f^{2}(y) d y\right)^{1 / 2}$, and the constant $N$ depends on $K, t, m$, and $\alpha$ only.

The estimates of the form (1.2) are important in the theory of stochastic differential equations as well as in their applications such as control theory, nonlinear filtering, etc. The above estimates are called Krylov's estimates because N. V. Krylov was the first who proved them for processes $X$ of diffusion type [KR], the case when $\alpha=2$. Some generalizations of Krylov's estimates for diffusion processes

Received by the editors October 4, 2005 and, in revised form, December 1, 2005.

2000 Mathematics Subject Classification. Primary 60H10, 60J60, 60J65, 60G44.

Key words and phrases. One-dimensional stochastic equations, bounded drift, Krylov's estimates, weak convergence, symmetric stable processes. 
with jumps were obtained by S. Anulova and H. Pragarauskas [AP], and J. P. Lepeltier and B. Marchal $[\mathrm{LM}]$. We refer also to $[\mathrm{M}]$ where one derived the estimates of the form (1.2) for some classes of semimartingales $X$.

H. Pragarauskas $[\mathrm{PR}]$ proved a variant of Krylov's estimates for processes $X$ of the form (1.1) without drift $(a=0)$ and $\alpha \in(1,2)$. More precisely, he proved that, for any $m \in \mathbb{N}, t \geq 0$ and any measurable function $f:[0, \infty) \times \mathbb{R} \rightarrow[0, \infty)$, it holds that

$$
\mathbf{E} \int_{0}^{t \wedge \tau_{m}(X)}\left|b_{s}\right|^{\alpha / 2} f\left(s, X_{s}\right) d s \leq N\|f\|_{2, m, t}
$$

where $\|f\|_{2, m, t}=:\left(\int_{0}^{t} \int_{-m}^{m} f^{2}(s, y) d s d y\right)^{1 / 2}$ and the constant $N$ depends on $m, t$, and $\alpha$ only.

As an application of the estimates (1.2), we prove the existence of solutions of the time-independent stochastic differential equation

$$
d X_{t}=b\left(X_{t-}\right) d Z_{t}+a\left(X_{t}\right) d t, \quad X_{0}=x_{0} \in \mathbb{R}, \quad t \geq 0,
$$

where $Z$ is a symmetric stable process of index $\alpha \in(1,2)$ with $Z_{0}=0$ and $a, b$ : $\mathbb{R} \rightarrow \mathbb{R}$ are measurable functions. We require the diffusion coefficient $b$ to be nondegenerate and bounded and the drift $a$ to be bounded.

If $\alpha=2$, then $Z$ is a Brownian motion process and the equation (1.3) becomes the Itô equation. In the case of equation (1.3) without drift $(a=0)$, there are known necessary and sufficient conditions for the existence and uniqueness of solutions found by H. J. Engelbert and W. Schmidt [ES2]. The case of equation (1.3) with drift was considered in [ES1] where one found sufficient conditions for the existence of (in general, exploding) solutions by using the well-known Zvonkin transformation.

For $\alpha \neq 2$ the process $Z$ is a purely discontinuous Levy process. Moreover, it is a semimartingale (in particular, a martingale for $\alpha>1$ ) so that the stochastic integral in (1.3) can be understood in the sense of stochastic integration with respect to a semimartingale. The equation (1.3) without drift $(a=0)$ was investigated by P. A. Zanzotto [Z2]. He completely generalized the results of Engelbert and Schmidt for the case $1<\alpha<2$ and obtained some sufficient existence conditions for $\alpha \in(0,1]$.

A particular case of (1.3), the equation

$$
d X_{t}=d Z_{t}+a\left(X_{t}\right) d t, \quad X_{0}=x_{0} \in \mathbb{R}, \quad t \geq 0,
$$

was considered in $[\mathrm{TTW}]$ where one required the coefficient $a$ to be continuous and bounded. More recently, N. I. Portenko [PO] was able to construct a solution of the equation (1.4) with $\alpha \in(1,2)$ assuming that there exists a number $p>(\alpha-1)^{-1}$ such that $\left(\int_{\mathbb{R}}|a|^{p}(y) d y\right)^{1 / p}<\infty$. The method used by Portenko was a purely analytical one and based on some estimates for the transition probability density of a symmetric stable process. Here we use a probabilistic approach that relies on a version of Krylov's estimates for stable integrals with drift and the technique of weak convergence for cádlag processes. We also notice that Portenko's condition allows the drift $a$ to be unbounded but globally Lebesgue integrable of the required power $p$. On the other hand, it doesn't guarantee the existence of solutions for SDE's with an arbitrary bounded drift $a$ because a bounded function doesn't need to be Lebesgue integrable on $\mathbb{R}$. In this note we prove the existence of solutions for equation (1.4) when the drift $a$ is bounded. 
The paper is organized as follows. Section 2 collects some preliminary facts about symmetric stable processes and discusses the time change method for stochastic equations with drift driven by symmetric stable processes. In section 3 we deal with so-called Krylov's estimates for stochastic integrals with drift. There we solve the Bellman equation associated with the corresponding optimization problem controlled by a stable integral with drift, and derive an $L_{2}$-estimate for the solution. Section 3 closes with the proof of $L_{2}$-estimates for processes of the form (1.1). Finally, as an application of the derived Krylov's estimates, we prove in section 4 the existence of solutions of equations (1.3) and (1.4) for $\alpha \in(1,2)$.

\section{PRELIMINARY REMARKS}

In this section we assume $0<\alpha \leq 2$ and begin with some definitions. As usual, we denote by $\mathbb{D}_{[0, \infty)}(\mathbb{R})$ the Skorokhod space, i.e. the set of all real-valued functions $\omega:[0, \infty) \rightarrow \mathbb{R}$ with right-continuous trajectories and with finite left limits (also called cádlag functions). For simplicity, we shall write $\mathbb{D}$ instead of $\mathbb{D}_{[0, \infty)}(\mathbb{R})$. We will equip $\mathbb{D}$ with the $\sigma$-algebra $\mathcal{D}$ generated by the Skorokhod topology. Under $\mathbb{D}^{n}$ we will understand the $n$-dimensional Skorokhod space defined as $\mathbb{D}^{n}=\mathbb{D} \times \cdots \times \mathbb{D}$ with the corresponding $\sigma$-algebra $\mathcal{D}^{n}$ being the direct product of $n$ one-dimensional $\sigma$-algebras $\mathcal{D}$.

Let $(\Omega, \mathcal{F}, \mathbf{P})$ be a complete probability space carrying a process $Z$ with $Z_{0}=0$ and let $\mathbf{F}=\left(\mathcal{F}_{t}\right)$ be a filtration on $(\Omega, \mathcal{F}, \mathbf{P})$. The notation $(Z, \mathbf{F})$ means that $Z$ is adapted to the filtration $\mathbf{F}$. We call $(Z, \mathbf{F})$ a symmetric stable process of index $\alpha \in(0,2]$ if trajectories of $Z$ belong to $\mathbb{D}$ and $\mathbf{E}\left(\exp \left(i \xi\left(Z_{t}-Z_{s}\right)\right) \mid \mathcal{F}_{s}\right)=$ $\exp \left(-(t-s)|\xi|^{\alpha}\right)$ for all $t>s \geq 0$ and $\xi \in \mathbb{R}$.

A stochastic process $(X, \mathbf{F})$, defined on a probability space $(\Omega, \mathcal{F}, \mathbf{P})$ with a filtration $\mathbf{F}=\left(\mathcal{F}_{t}\right)_{t \geq 0}$ and with trajectories in $\mathbb{D}$, is called a (weak) solution of the equation (1.3) with initial value $x_{0} \in \mathbb{R}$ if there exists a symmetric stable process $(Z, \mathbf{F})$ such that $Z_{0}=0$ and

$$
X_{t}=x_{0}+\int_{0}^{t} b\left(X_{s-}\right) d Z_{s}+\int_{0}^{t} a\left(X_{s}\right) d s, \quad t \geq 0 \quad \text { P-a.s. }
$$

As mentioned above, for all $0<\alpha \leq 2$, a symmetric stable process $Z$ is a semimartingale so that the stochastic integral in (2.1) can be defined for all appropriate integrands via semimartingale integration theory.

Because $Z$ is a process with independent increaments, it is obviously a Markov process. Therefore, it can be characterized in terms of Markov processes. For any function $f \in L^{\infty}(\mathbb{R})$ and $t \geq 0$, define the operator

$$
\left(P_{t} f\right)(x):=\int_{\Omega} f\left(x+Z_{t}\right) d \mathbf{P}(\omega)
$$

where $L^{\infty}(\mathbb{R})$ is the Banach space of functions $f: \mathbb{R} \rightarrow \mathbb{R}$ with the norm $\|f\|_{\infty}=$ ess sup $|f(x)|$. The family $\left(P_{t}\right)_{t \geq 0}$ is called the family of convolution operators associated with $Z$. Formally, for a suitable class of functions $g(x)$, we can define the so-called infinitesimal generator $\mathcal{A}$ of the process $Z$ as

$$
(\mathcal{A} g)(x)=\lim _{t \downarrow 0} \frac{\left(P_{t} g\right)(x)-g(x)}{t} .
$$

On another hand, in the case of $\alpha \in(0,2), Z$ is a purely discontinuous Markov process that can be described by its Poisson jump measure (jump measure of $Z$ on 
the interval $[0, t])$ defined as

$$
\mu(U \times[0, t])=\sum_{s \leq t} 1_{U}\left(Z_{s}-Z_{s-}\right),
$$

the number of times before the time $t$ that $Z$ has jumps whose size lies in the set $U$. The compensating measure of $\mu$, say $\nu$, is given (see, e.g. [K], Prop. 13.9) by

$$
\nu(U)=\mathbf{E} \mu(U \times[0,1])=\int_{U} \frac{1}{|x|^{1+\alpha}} d x .
$$

It is known that for $\alpha<2$

$$
(\mathcal{A} g)(x)=\int_{\mathbb{R} \backslash\{0\}}\left[g(x+z)-g(x)-\mathbf{1}_{\{|z|<1\}} g^{\prime}(x) z\right] \frac{c_{\alpha}}{|z|^{1+\alpha}} d z
$$

for any $g \in C^{2}$, where $C^{2}$ is the set of all bounded and twice continuously differentiable functions $g: \mathbb{R} \rightarrow \mathbb{R}$ and $c_{\alpha}$ is a suitable constant. Contrary to the case of $\alpha \in(0,2)$, the infinitesimal generator of a Brownian motion process $(\alpha=2)$ is the Laplacian, that is, the second derivative operator.

It can be noticed that the use of Fourier transform may simplify the calculations essentially when working with the infinitesimal generator $\mathcal{A}$. Let $g \in L_{1}(\mathbb{R})$ and

$$
F g(x):=\int_{\mathbb{R}} e^{i z x} g(z) d z
$$

be the Fourier transform of $g$. The following facts will be used later.

Proposition 2.1. Let $0<\alpha \leq 2$ and let $\left(P_{t}\right)$ and $\mathcal{A}$ be the operators as defined above associated with the symmetric stable process $Z$. The following statements are true:

(i) For every function $g \in L^{\infty} \cap L_{1}$, it holds that

$$
F\left(P_{t} g\right)(x)=e^{-t|x|^{\alpha}} F g(x) \text {. }
$$

(ii) Assume that $g \in C^{2}$ and $\mathcal{A} g \in L_{1}$. Then, it holds that

$$
F(\mathcal{A} g)(x)=-|x|^{\alpha} F g(x) .
$$

Proof. The proof is straightforward. We have for (i):

$$
\begin{gathered}
F\left(P_{t} g\right)(x)=\int_{\mathbb{R}} e^{i x z} P_{t} g(z) d z=\mathbf{E}\left(\int_{\mathbb{R}} e^{i x z} g\left(Z_{t}+z\right) d z\right) \\
=\mathbf{E}\left(\int_{\mathbb{R}} e^{i x\left(y-Z_{t}\right)} g(y) d y\right)=\mathbf{E} e^{-i x Z_{t}} \int_{\mathbb{R}} e^{i x y} g(y) d y=e^{-t|x|^{\alpha}} F g(x) .
\end{gathered}
$$

The statement (ii) follows from (i) and the definition (2.2):

$$
\begin{gathered}
F(\mathcal{A} g)(x)=\int_{\mathbb{R}} e^{i x z} \mathcal{A} g(z) d z=\int_{\mathbb{R}} e^{i x z} \lim _{t \downarrow 0} \frac{P_{t} g(z)-g(z)}{t} d z \\
=\lim _{t \downarrow 0} \frac{1}{t}\left(\int_{\mathbb{R}} e^{i x z} P_{t} g(z) d z-\int_{\mathbb{R}} e^{i x z} g(z) d z\right)=\lim _{t \downarrow 0} \frac{1}{t}\left(e^{-t|x|^{\alpha}} F g(x)-F g(x)\right) \\
=F g(x) \lim _{t \downarrow 0} \frac{e^{-t|x|^{\alpha}}-1}{t}=-|x|^{\alpha} F g(x) .
\end{gathered}
$$


Let $\alpha \in(1,2), f \in C^{2}$, and define

$$
Y_{t}=\int_{0}^{t} h_{s} d Z_{s}
$$

where $h$ lies in a suitable class of integrands so that the stochastic integral exists. It then follows (see [B], Proposition 2.1) that

$$
f\left(Y_{t}\right)=f\left(Y_{0}\right)+M_{t}+\int_{0}^{t}\left|h_{s}\right|^{\alpha} \mathcal{A} f\left(Y_{s-}\right) d s,
$$

where $M$ is a martingale.

Finally, let us point out how one can construct a solution of the equation (1.3) for any $\alpha \in(0,2]$ using the time change method. Recall first that a process $A$ is called a $\mathbf{F}$-time change if it is an increasing right-continuous process with $A_{0}=0$ such that $A_{t}$ is a $\mathbf{F}$-stopping time for any $t \geq 0$ (cf. [K], chapter 6). Define $T_{t}=: \inf \left\{s \geq 0: A_{s}>t\right\}$ called the right-continuous inverse process to $A$. By definition, $T$ is an increasing process starting at zero. It is easy to see that $T$ is a $\mathbf{F}$-adapted process if and only if $A$ is a $\mathbf{F}$-time change.

Let $\bar{Z}$ be any symmetric stable process of index $\alpha \in(0,2], x_{0} \in \mathbb{R}$ is any initial value and consider the equation

$$
Y_{t}-x_{0}=\bar{Z}_{t}+\int_{0}^{t} a|b|^{-\alpha}\left(Y_{s}\right) d s
$$

where $|b|^{-\alpha}=1 /|b|^{\alpha}$.

Proposition 2.2. Assume that there exist constants $\delta_{1}>0$ and $\delta_{2}>0$ such that $\delta_{1} \leq|b| \leq \delta_{2}$. Then, the equation (1.3) has a solution if and only if the equation (2.5) has a solution.

Proof. Suppose first that $X$ is a solution of the equation (1.3) which means that the equation (2.1) is satisfied. The integrals on the right side of (2.1) are well-defined and are $\mathbf{P}$-a.s. finite for all $t \geq 0$. Let

$$
A_{t}=\int_{0}^{t}|b|^{\alpha}\left(X_{s}\right) d s
$$

and

$$
T_{t}=\inf \left\{s \geq 0: A_{s}>t\right\} .
$$

In can be easily verified that the process $T$ satisfies the relation

$$
T_{t}=\int_{0}^{t}|b|^{-\alpha}\left(X_{T_{s}}\right) d s
$$

By definition, the process $A$ is $\mathbf{F}$-adapted so that its right-inverse process $T$ is a F-time change process defined for all $t \geq 0$. We notice that $\left(T_{t}\right)$ is a global time change $^{1}$ because $A_{\infty}=\lim _{t \uparrow \infty} A_{t}=\infty$. Now define

$$
Y_{t}=X_{T_{t}}, \quad \mathcal{G}_{t}=\mathcal{F}_{T_{t}} \text {. }
$$

Applying the time change $t \rightarrow T_{t}$ to the semimartingale $X$ in $(2.1)$ (see [J], Chapter 10) and using the change of variables rule in Lebesgue-Stieltjes integral (see ch. 0 , (4.9) in $[R Y]$ ) yields

$$
Y_{t}=x_{0}+\int_{0}^{T_{t}} b\left(X_{s-}\right) d Z_{s}+\int_{0}^{t} a\left(Y_{s}\right) d T_{s} .
$$

\footnotetext{
${ }^{1}$ That is, $T_{t} \in[0, \infty)$ for all $t \geq 0$.
} 
It remains to notice that the process

$$
\bar{Z}_{t}:=\int_{0}^{T_{t}} b\left(X_{s-}\right) d Z_{s}
$$

is nothing but a symmetric stable process of the index $\alpha$ (see [RW], Theorem 3.1). Hence $Y$ is a solution of the equation (2.5).

The proof of the opposite direction is a very similar one. For this, suppose that the process $Y$ is a solution of the equation (2.5) defined on a probability space $(\Omega, \mathcal{G}, \mathbf{P})$ with a filtration $\mathbf{G}$ where $\bar{Z}$ is a symmetric stable process adapted to $\mathbf{G}$. Define

$$
T_{t}=\int_{0}^{t}|b|^{-\alpha}\left(Y_{s}\right) d s
$$

and let

$$
X_{t}=Y_{A_{t}}, \mathcal{F}_{t}=\mathcal{G}_{A_{t}}
$$

for all $t \geq 0$ where $A$ is the right inverse to $T$ and $T_{\infty}=\lim _{t \uparrow \infty} T_{t}=\infty$. By applying the global time change $t \rightarrow A_{t}$ to the semimartingale $Y$ in (2.5) we obtain

$$
\bar{Z}_{A_{t}}=X_{t}-x_{0}-\int_{0}^{t} a\left(X_{s}\right) d s .
$$

Let $R_{t}=\bar{Z}_{A_{t}}, t \geq 0$, and first consider the case $0<\alpha<2$. According to the properties of time change in semimartingales (see, e.g. [J], Chapter 10), $R$ is a purely discontinuous martingale with the jump measure $\mu_{R}(d t, d x)$ and its compensator (dual predictable projection of $\left.\mu_{R}\right) \nu_{R}(d t, d x)$. It follows from (2.3) that the compensator of a symmetric stable process of index $\alpha$ has the form

$$
\nu_{\bar{Z}}(d t, d x)=d t \times \frac{d x}{|x|^{\alpha+1}} .
$$

Applying a continuous time change to a purely discontinuous martingale gives a purely discontinuous martingale with the compensator obtained from that of the original process. We then have

$$
\nu_{R}(d t, d x)=d A_{t} \times \frac{d x}{|x|^{\alpha+1}}=|b|^{\alpha}\left(X_{t}\right) d t \times \frac{d x}{|x|^{\alpha+1}} .
$$

Hence $R$ is a purely discontinuous martingale with the compensator (2.6) that is absolute continuous with respect to the compensator of a symmetric stable process of index $\alpha$. The representation theorem for symmetric stable processes (see [Z1]) yields that there exists a symmetric stable process $Z$ defined on the same probability space such that

$$
R_{t}=\int_{0}^{t} b\left(X_{s-}\right) d Z_{s}
$$

If $\alpha=2$, then the process $\bar{Z}_{A_{t}}$ is a continuous local martingale with the quadratic variation process $\langle\bar{Z}\rangle_{t}=A_{t}=\int_{0}^{t} b^{2}\left(X_{s}\right) d s$ so that after applying the classical representation theorem of Doob (see, e.g. [IW], Ch. 2, Theorem 7.1) we conclude that there exists a Brownian motion $Z$ such that (2.7) holds as well. Thus we have shown that $X$ is a solution of the equation (1.3). 


\section{3. $L_{2}$-ESTimates FOR STABle INTEGRALS With DRIFT}

In this section we are first going to derive an $L_{2}$-estimate for solutions of a given class of quasilinear partial differential equtions. Then, based on that result, we shall prove some of Krylov's estimates for stable integrals with drift that will play a key role in the forthcoming existence theorems.

Let $K>0$ be a constant and $f$ be a nonnegative, measurable function such that $f \in C_{0}^{\infty}(\mathbb{R})$, where $C_{0}^{\infty}(\mathbb{R})$ denotes the class of all infinitely differentiable real valued functions with compact support defined on $\mathbb{R}$. Suppose further that $Z$ is a symmetric stable process of index $\alpha \in(1,2)$ defined on a probability space $(\Omega, \mathcal{F}, \mathbf{P})$ with filtration $\mathbf{F}$. By $\mathcal{T}$ we denote the class of all $\mathbf{F}$-predictable processes $\left(\gamma_{t}\right)$ such that $\left|\gamma_{t}\right| \leq K$.

Consider the controlled processes $X^{\gamma}$ defined as

$$
d X_{t}^{\gamma}=d Z_{t}+\gamma_{t} d t
$$

and, for any $\lambda>0$, define the corresponding value function $v(x), x \in \mathbb{R}$, by

$$
v(x)=\sup _{\gamma \in \mathcal{T}} \mathbf{E} \int_{0}^{\infty} e^{-\lambda t} f\left(x+X_{t}^{\gamma}\right) d t .
$$

The Bellman principle of optimality can be formulated for the controlled processes $X^{\gamma}$ and the value function $v$ as follows:

For any $\mathbf{F}$-stopping time $\tau$ it holds that

$$
v(x)=\sup _{\gamma \in \mathcal{T}} \mathbf{E}\left\{\int_{0}^{\tau} e^{-\lambda t} f\left(x+X_{t}^{\gamma}\right) d t+e^{-\lambda \tau} v\left(x+X_{\tau}^{\gamma}\right)\right\} .
$$

Using standard arguments, one can derive from the principle above the corresponding Bellman equation ( $\gamma$ is deterministic)

$$
\sup _{\gamma \leq K}\left\{\mathcal{A} v(x)-\lambda v(x)+\gamma v_{x}(x)+f(x)\right\}=0
$$

which holds a.e. in $\mathbb{R}$ and $v_{x}$ denotes the first derivative of $v$. It is easy to see that the Bellman equation is equivalent to the equation

$$
\mathcal{A} v-\lambda v+K\left|v_{x}\right|+f=0 .
$$

Now, for any measurable function $h: \mathbb{R} \rightarrow \mathbb{R}$, define $\|h\|_{2}:=\left(\int_{\mathbb{R}} h^{2}(x) d x\right)^{1 / 2}$ to be the $L_{2}$-norm of $h$.

Lemma 3.1. For all $x \in \mathbb{R}$, it holds that

$$
v(x) \leq N\|f\|_{2},
$$

where the constant $N$ depends on $K$ and $\alpha$ only.

Proof. Let $q(x)$ be a nonnegative function such that $q \in C_{0}^{\infty}(\mathbb{R})$ and $\int_{\mathbb{R}} q(x) d x=1$. For any function $h: \mathbb{R} \rightarrow[0, \infty)$ and any $\varepsilon>0$ let

$$
h^{(\varepsilon)}(x)=\frac{1}{\varepsilon} \int_{\mathbb{R}} q\left(\frac{x-y}{\varepsilon}\right) h(y) d y
$$

be the $\varepsilon$-convolution of $h$ with $q$. For any $\varepsilon>0$, define

$$
f^{(\varepsilon)}=-\mathcal{A} v^{(\varepsilon)}+\lambda v^{(\varepsilon)}-K\left|v_{x}^{(\varepsilon)}\right| .
$$

Clearly, $f^{(\varepsilon)}$ is square integrable and $f^{(\varepsilon)} \rightarrow f$ a.s. as $\varepsilon \rightarrow 0$. 
It follows from (3.3) that

$$
\left(\mathcal{A} v^{(\varepsilon)}-\lambda v^{(\varepsilon)}\right)^{2}=\left(K\left|v_{x}^{(\varepsilon)}\right|+f^{(\varepsilon)}\right)^{2}
$$

and

$$
\begin{gathered}
\int_{\mathbb{R}}\left(\mathcal{A} v^{(\varepsilon)}(x)-\lambda v^{(\varepsilon)}(x)\right)^{2} d x=\int_{\mathbb{R}}\left(K\left|v_{x}^{(\varepsilon)}\right|(x)+f^{(\varepsilon)}(x)\right)^{2} d x \\
\leq 2 K^{2} \int_{\mathbb{R}}\left(v_{x}^{(\varepsilon)}(x)\right)^{2} d x+2 \int_{\mathbb{R}}\left(f^{(\varepsilon)}(x)\right)^{2} d x .
\end{gathered}
$$

Using Proposition 2.1, the Parseval-Plancherel equality

$$
\int_{\mathbb{R}}\left(v^{(\varepsilon)}(x)\right)^{2} d x=\frac{1}{2 \pi} \int_{\mathbb{R}}\left|F v^{(\varepsilon)}(z)\right|^{2} d z,
$$

and integration by parts, one obtains from (3.4) that

$$
\int_{\mathbb{R}}\left|F v^{(\varepsilon)}(x)\right|^{2}\left(|x|^{\alpha}+\lambda\right)^{2} d x \leq 2 K^{2} \int_{\mathbb{R}}\left|F v^{(\varepsilon)}(x)\right|^{2}|x|^{2} d x+2 \int_{\mathbb{R}}\left(F f^{(\varepsilon)}(x)\right)^{2} d x .
$$

It is easy to see that there exists a constant $\mu>0$ such that

$$
\left(|x|^{\alpha}+\mu\right)^{2} \geq 4 K^{2}|x|^{2}
$$

for all $x \in \mathbb{R}$.

The inequalities (3.5) and (3.6) combined yield for all $\lambda \geq \mu$

$$
\frac{1}{2} \int_{\mathbb{R}}\left|F v^{(\varepsilon)}(x)\right|^{2}\left(|x|^{\alpha}+\lambda\right)^{2} d x \leq 2 \int_{\mathbb{R}}\left(F f^{(\varepsilon)}(x)\right)^{2} d x .
$$

Applying the inverse Fourier transform and the Cauchy-Schwarz inequality, we finally obtain for all $y \in \mathbb{R}$ and $\lambda \geq \mu$

$$
\leq \frac{1}{4 \pi^{2}} \int_{\mathbb{R}}\left|F v^{(\varepsilon)}(x)\right|^{2}\left(|x|^{\alpha}+\lambda\right)^{2} d x \int_{\mathbb{R}}\left(|x|^{\alpha}+\lambda\right)^{2} d x \leq N \int_{\mathbb{R}}\left(f^{(\varepsilon)}(x)\right)^{2} d x,
$$

where

$$
N=\frac{1}{\pi^{2}} \int_{\mathbb{R}}\left(|x|^{\alpha}+\lambda\right)^{-2} d x .
$$

The desired estimate then follows by taking the limit $\varepsilon \rightarrow 0$ in the above inequality and using the Lebesgue dominated convergence theorem.

Now, let $X$ be a solution of the equation (1.3). We are interested in $L_{2}$ - estimates of the form

$$
\mathbf{E} \int_{0}^{\infty} e^{-\lambda \psi_{u}} \varphi_{u} f\left(x+X_{u}\right) d u \leq N\|f\|_{2},
$$

where $\psi$ and $\varphi$ are some nonnegative predictable processes. We will assume that

$$
|a(x)| \leq K|b(x)|^{\alpha} \quad \text { for all } \quad x \in \mathbb{R} .
$$


Theorem 3.2. Suppose $X$ is a solution of the equation (1.3) with $\alpha \in(1,2)$ and the condition (3.7) is satisfied. Then, for any $x \in \mathbb{R}, \lambda \geq \mu$, and any measurable function $f: \mathbb{R} \rightarrow[0, \infty)$, it holds that

$$
\mathbf{E} \int_{0}^{\infty} e^{-\lambda \psi_{u}}\left|b\left(X_{u}\right)\right|^{\alpha} f\left(x+X_{u}\right) d u \leq N\|f\|_{2},
$$

where $\psi_{t}=\int_{0}^{t}\left|b\left(X_{s}\right)\right|^{\alpha} d s$ and the constant $N$ depends on $K$ and $\alpha$ only.

Proof. Assume first that $f \in C_{0}^{\infty}(\mathbb{R})$ so that there is a solution $v$ of equation (3.1) satisfying the inequality (3.2). By taking the $\varepsilon$-convolution on both sides of (3.1), we obtain

$$
\mathcal{A} v^{(\varepsilon)}-\lambda v^{(\varepsilon)}+K\left|v_{x}^{(\varepsilon)}\right|+f^{(\varepsilon)} \leq 0 .
$$

Then, for all $x \in \mathbb{R}$, applying Itô's formula (cf. (2.4)) to the expression

$$
v^{(\varepsilon)}\left(x+X_{s}\right) e^{-\lambda \psi_{s}},
$$

yields

$$
\begin{gathered}
\mathbf{E} v^{(\varepsilon)}\left(x+X_{s}\right) e^{-\lambda \psi_{s}}-v^{(\varepsilon)}(x) \\
=\mathbf{E} \int_{0}^{s} e^{-\lambda \psi_{u}}\left[\left|b\left(X_{u}\right)\right|^{\alpha} \mathcal{A} v^{(\varepsilon)}-\left|b\left(X_{u}\right)\right|^{\alpha} \lambda v^{(\varepsilon)}+a\left(X_{u}\right) v_{x}^{(\varepsilon)}\right]\left(x+X_{u}\right) d u \\
\leq \mathbf{E} \int_{0}^{s} e^{-\lambda \psi_{u}}\left|b\left(X_{u}\right)\right|^{\alpha}\left[\mathcal{A} v^{(\varepsilon)}-\lambda v^{(\varepsilon)}+K\left|v_{x}^{(\varepsilon)}\right|\right]\left(x+X_{u}\right) d u \\
\leq-\mathbf{E} \int_{0}^{s} e^{-\lambda \psi_{u}}\left|b\left(X_{u}\right)\right|^{\alpha} f^{(\varepsilon)}\left(x+X_{u}\right) d u .
\end{gathered}
$$

Hence using Lemma 3.1 we obtain

$$
\mathbf{E} \int_{0}^{s} e^{-\lambda \psi_{u}}\left|b\left(X_{u}\right)\right|^{\alpha} f^{(\varepsilon)}\left(x+X_{u}\right) d u \leq \sup _{x} v^{(\varepsilon)}(x) \leq N\left\|f^{(\varepsilon)}\right\|_{2} .
$$

Letting $\varepsilon \rightarrow 0$ and $s \rightarrow \infty$ and using the Fatou's lemma, we arrive at

$$
\mathbf{E} \int_{0}^{\infty} e^{-\lambda \psi_{u}}\left|b\left(X_{u}\right)\right|^{\alpha} f\left(x+X_{u}\right) d u \leq N\|f\|_{2} .
$$

The latter inequality can be extended in a standard way first to any function $f \in$ $L_{2}(\mathbb{R})$ and then to any nonnegative, measurable function using the monotone class theorem arguments (see, for example, [DM], Theorem 20).

Corollary 3.3. Suppose $X$ is a solution of the equation (1.4) with $\alpha \in(1,2)$ and $|a(x)| \leq K$. Then, for any $t \geq 0$ and any measurable function $f: \mathbb{R} \rightarrow[0, \infty)$, it holds that

$$
\mathbf{E} \int_{0}^{t} f\left(X_{u}\right) d u \leq N\|f\|_{2}
$$

where the constant $N$ depends on $K, t$, and $\alpha$ only.

Proof. The desired estimate follows immediately from Theorem 3.2 for $b=1$.

One can also easily obtain the local version of the estimate (3.8). For arbitrary but fixed $m \in \mathbb{N}$, define $\|f\|_{2, m}=\left(\int_{[-m, m]}|f(x)|^{2} d x\right)^{\frac{1}{2}}$ as the $L_{2}$-norm of $f$ on $[-m, m]$. 
Applying (3.8) to the function $\bar{f}(y)=f(y) \mathbf{1}_{[-m, m]}(y)$, we obtain

Corollary 3.4. Let $X$ be a solution of the equation (1.3) with $\alpha \in(1,2)$ and let the condition (3.7) be satisfied. Then, for any $t \geq 0, m \in \mathbb{N}$, and any nonnegative measurable function $f$, it holds that

$$
\mathbf{E} \int_{0}^{t \wedge \tau_{m}(X)}\left(|b|^{\alpha} f\right)\left(X_{u}\right) d u \leq N\|f\|_{2, m},
$$

where $N$ is a constant depending on $K, \alpha, m$, and $t$ only.

Clearly, the corresponding estimate from Corollary 3.3 also follows for the local Krylov's estimate of the form (3.9).

Corollary 3.5. Let $X$ be a solution of the equation (1.4) with $\alpha \in(1,2)$ and $|a(x)| \leq K$. Then, for any $t \geq 0, m \in \mathbb{N}$, and any nonnegative measurable function $f$, it holds that

$$
\mathbf{E} \int_{0}^{t \wedge \tau_{m}(X)} f\left(X_{u}\right) d u \leq N\|f\|_{2, m},
$$

where $N$ is a constant depending on $K, \alpha, m$, and $t$ only.

\section{Existence OF SOlutions FOR SDE'S With BOUNDED DRIFT}

Here we are going to apply the estimates derived in section 3 to prove the existence of solutions of stochastic equations with drift. We shall prove the existence of weak solutions of the equation (1.3) for measurable coefficients $a$ and $b$ such that $a$ is bounded and $b$ is nondegenerate and bounded. More precisely, we assume that there exists a constant $\Lambda>0$ such that

1) $\frac{1}{\Lambda} \leq|b(x)| \leq \Lambda, \quad|a(x)| \leq \Lambda$ for all $x \in \mathbb{R}$.

For $\alpha=2$, the existence of weak solutions under the condition $\boldsymbol{\Lambda}$ ) is well-known (cf. [ES1]). Hencefore, we restrict ourself to the case $1<\alpha<2$.

Theorem 4.1. Suppose that the coefficients a and b satisfy the assumption $\boldsymbol{\Lambda})$ and $\alpha \in(1,2)$. Then, for any $x_{0} \in \mathbb{R}$, there exists a solution of the equation (1.3).

Proof. According to Proposition 2.2, it suffices to prove that the equation

$$
d X_{t}=d Z_{t}+\sigma\left(X_{t}\right) d t, \quad X_{0}=x_{0} \in \mathbb{R}, \quad t \geq 0,
$$

has a solution where $\sigma=a|b|^{-\alpha}$ such that $|\sigma| \leq \Lambda^{1+\alpha}:=\Lambda_{1}$.

Using the standard arguments (see, for example, $[\mathrm{KR}]$ ), for $n=1,2, \ldots$, we can define the sequences of functions $\sigma_{n}(x)$ such that they are globally Lipshitz continuous, uniformly bounded by the constant $\Lambda_{1}$, and $\sigma_{n} \rightarrow \sigma$ uniformly as $n \rightarrow \infty$. Then, for any $n=1,2, \ldots$, the equation (4.1) has a unique solution, even a so-called strong solution (see, for example, Theorem 9.1 in [IW]). That is, for any fixed symmetric stable process $Z$ defined on a probability space $(\Omega, \mathcal{F}, \mathbf{P})$, there exists a sequence of processes $X^{n}, n=1,2, \ldots$, such that

$$
X_{t}^{n}=x_{0}+Z_{t}+\int_{0}^{t} \sigma_{n}\left(X_{s}^{n}\right) d s, \quad t \geq 0, \quad \mathbf{P} \text {-a.s. }
$$

Define

$$
Y_{t}^{n}=\int_{0}^{t} \sigma_{n}\left(X_{s}^{n}\right) d s
$$


so that

$$
X^{n}=x_{0}+Z+Y^{n}, \quad n \geq 1 .
$$

Now we are going to show that the sequence of 3-dimensional processes $H^{n}:=$ $\left(X^{n}, Y^{n}, Z\right), n \geq 1$, is tight in the sense of weak convergence in $\left(\mathbb{D}^{3}, \mathcal{D}^{3}\right)$. Due to the well-known Aldous' criterion ([A]), it suffices to show that

$$
\lim _{l \rightarrow \infty} \limsup _{n \rightarrow \infty} \mathbf{P}\left[\sup _{0 \leq s \leq t}\left\|H_{s}^{n}\right\|>l\right]=0
$$

for all $t \geq 0$ and

$$
\limsup _{n \rightarrow \infty} \mathbf{P}\left[\left\|H_{t \wedge\left(\tau^{n}+\delta_{n}\right)}^{n}-H_{t \wedge \tau^{n}}^{n}\right\|>\varepsilon\right]=0
$$

for all $t \geq 0, \varepsilon>0$, every sequence of $\mathbf{F}$-stopping times $\tau^{n}$, and every sequence of real numbers $\delta_{n}$ such that $\delta_{n} \downarrow 0$. Here $\|\cdot\|$ denotes the Euclidean norm of a vector.

It is clear that for this it suffices only to verify that the sequence of processes $Y^{n}$ is tight in $(\mathbb{D}, \mathcal{D})$. But this is trivially fulfilled because of the uniform boundness of the coefficients $\sigma_{n}, n \geq 1$.

From the tightness of the sequence $\left\{H^{n}\right\}$ we conclude that there exists a subsequence $\left\{n_{k}\right\}, k=1,2, \ldots$, a probability space $(\bar{\Omega}, \overline{\mathcal{F}}, \overline{\mathbf{P}})$ and the process $\bar{H}$ on it with values in $\left(\mathbb{D}^{3}, \mathcal{D}^{3}\right)$ such that $H^{n_{k}}$ converges weakly (in distribution) to the process $\bar{H}$ as $k \rightarrow \infty$. For simplicity, let $\left\{n_{k}\right\}=\{n\}$.

Next we use the famous embedding principle of Skorokhod (see, e.g. Theorem 2.7 in [IW]) to imply the convergence of the sequence $\left\{H^{n}\right\}$ a.s. in the following sense: there exists a probability space $(\tilde{\Omega}, \tilde{\mathcal{F}}, \tilde{\mathbf{P}})$ and processes $\tilde{H}=(\tilde{X}, \tilde{Y}, \tilde{Z}), \quad \tilde{H}^{n}=$ $\left(\tilde{X}^{n}, \tilde{Y}^{n}, \tilde{Z}^{n}\right), \quad n=1,2, \ldots$, on it such that

1) $\tilde{H}^{n} \rightarrow \tilde{H}$ as $n \rightarrow \infty \tilde{\mathbf{P}}$-a.s.

2) $\tilde{H}^{n}=H^{n}$ in distribution for all $n=1,2, \ldots$

Using standard measurability arguments ([KR], chapter 2$)$, one can prove that the processes $\tilde{Z}^{n}$ and $\tilde{Z}$ are symmetric stable processes of the index $\alpha$ with respect to the augmented filtrations $\tilde{\mathbf{F}}^{n}$ and $\tilde{\mathbf{F}}$ generated by processes $\tilde{H}^{n}$ and $\tilde{H}$, respectively.

Relying on the above properties 1 ) and 2), and the equation (4.2), one can show ([KR], chapter 2) that

$$
\tilde{X}_{t}^{n}=x_{0}+\tilde{Z}_{t}^{n}+\int_{0}^{t} \sigma_{n}\left(\tilde{X}_{s}^{n}\right) d s, \quad t \geq 0, \quad \tilde{\mathbf{P}} \text {-a.s. }
$$

At the same time, from the properties 1),2) and the quasi-left continuity of the the processes $\tilde{X}^{n}$ it follows that

$$
\lim _{n \rightarrow \infty} \tilde{X}_{t}^{n}=\tilde{X}_{t}, \quad t \geq 0, \quad \tilde{\mathbf{P}}_{\text {-a.s. }}
$$

Hence in order to show that the process $\tilde{X}$ is a solution of the equation (4.1), it is enough to prove that, for all $t \geq 0$,

$$
\lim _{n \rightarrow \infty} \int_{0}^{t} \sigma_{n}\left(\tilde{X}_{s}^{n}\right) d s=\int_{0}^{t} \sigma\left(\tilde{X}_{s}\right) d s \quad \tilde{\mathbf{P}} \text { - a.s. }
$$

Now we remark that from the convergence in probability it follows that there is a subsequence for which the convergence with probability one holds. Therefore, to verify (4.5), it suffices to show that for all $t \geq 0$ and $\varepsilon>0$ we have

$$
\lim _{n \rightarrow \infty} \tilde{\mathbf{P}}\left[\left|\int_{0}^{t} \sigma_{n}\left(\tilde{X}_{s}^{n}\right) d s-\int_{0}^{t} \sigma\left(\tilde{X}_{s}\right) d s\right|>\varepsilon\right]=0 .
$$


We will need the following lemma.

Lemma 4.2. Let $\tilde{X}$ be the process as defined above. Then, for any Borel measurable function $f: \mathbb{R} \rightarrow[0, \infty)$ and any $t \geq 0$, there exists a sequence $m_{k} \in(0, \infty), k=$ $1,2, \ldots$, such that $m_{k} \uparrow \infty$ as $k \rightarrow \infty$ and it holds that

$$
\tilde{\mathbf{E}} \int_{0}^{t \wedge \tau_{m_{k}}(\tilde{X})} f\left(\tilde{X}_{s}\right) d s \leq N\|f\|_{2, m_{k}},
$$

where the constant $N$ depends on $\Lambda, \alpha, t$ and $m_{k}$ only.

Proof. Though the proof idea is essentially borrowed from [PZ], we carry out the proof for the convenience of the reader.

One proves first that there exists a sequence $m_{k} \in(0, \infty), k=1,2, \ldots$, with $m_{k} \uparrow \infty$ as $k \rightarrow \infty$ such that

$$
\lim _{n \rightarrow \infty} \tau_{m_{k}}\left(\tilde{X}^{n}\right)=\tau_{m_{k}}(\tilde{X}) \quad \tilde{\mathbf{P}} \text {-a.s. }
$$

It can be seen that the function $y \rightarrow \tau_{y}(z), y>0$, is increasing for any $z \in \mathbb{D}$. That means that $\tau_{y}(z)$ can have at most a countable number of points of discontinuity which we denote by $Q \in(0, \infty)$. Based on the proporties of the Skorokhod space $\mathbb{D}$, we conclude that if $z^{n} \rightarrow z$ as $n \rightarrow \infty$ in the Skorokhod topology, then there exists a sequence $m_{k} \in(0, \infty) \backslash Q$ such that

$$
\tau_{m_{k}}\left(z^{n}\right) \rightarrow \tau_{m_{k}}(z) \text { as } n \rightarrow \infty
$$

for all $m_{k} \in(0, \infty) \backslash Q$. Because of (4.4), we obtain (cf. Lemma 3.2 in [PZ])

$$
\tau_{m_{k}}\left(\tilde{X}^{n}\right) \rightarrow \tau_{m_{k}}(\tilde{X} \text {. }) \text { as } n \rightarrow \infty \quad \tilde{\mathbf{P}}_{\text {-a.s. }}
$$

Now suppose $f$ is a bounded and continuous function. Using Fatou's lemma, the Lebesgue bounded convergence theorem, and Krylov's estimate (3.10), we obtain

$$
\begin{gathered}
\tilde{\mathbf{E}} \int_{0}^{t \wedge \tau_{m_{k}}(\tilde{X})} f\left(\tilde{X}_{s}\right) d s=\tilde{\mathbf{E}} \int_{0}^{t} \liminf _{n \rightarrow \infty} \mathbf{1}_{\left\{s<\tau_{m_{k}}\left(\tilde{X}^{n}\right)\right\}} f\left(\tilde{X}_{s}^{n}\right) d s \\
=\liminf _{n \rightarrow \infty} \tilde{\mathbf{E}} \int_{0}^{t} \mathbf{1}_{\left\{s<\tau_{m_{k}}\left(\tilde{X}^{n}\right)\right\}} f\left(\tilde{X}_{s}^{n}\right) d s \leq N \liminf _{n \rightarrow \infty}\|f\|_{2, m_{k}}=N\|f\|_{2, m_{k}} .
\end{gathered}
$$

To finish the proof of the lemma it suffices to notice that the above estimate is also true for any Borel measurable function $f$ that can be verified by using the standard monotone class theorem arguments.

Without loss of generality, we can assume $\left\{m_{k}\right\}=\{m\}$.

Let us prove (4.6). For a fixed $k_{1} \in \mathbb{N}$ we have

$$
\begin{gathered}
\tilde{\mathbf{P}}\left[\left|\int_{0}^{t} \sigma_{n}\left(\tilde{X}_{s-}^{n}\right) d s-\int_{0}^{t} \sigma\left(\tilde{X}_{s-}\right) d s\right|>\varepsilon\right] \\
\leq \tilde{\mathbf{P}}\left[\left|\int_{0}^{t} \sigma_{k_{1}}\left(\tilde{X}_{s-}^{n}\right) d s-\int_{0}^{t} \sigma_{k_{1}}\left(\tilde{X}_{s-}\right) d s\right|>\frac{\varepsilon}{3}\right] \\
+\tilde{\mathbf{P}}\left[\left|\int_{0}^{t \wedge \tau_{m}\left(\tilde{X}^{n}\right)}\left[\sigma_{k_{1}}-\sigma_{n}\right]\left(\tilde{X}_{s-}^{n}\right) d s\right|>\frac{\varepsilon}{3}\right] \\
+\tilde{\mathbf{P}}\left[\left|\int_{0}^{t \wedge \tau_{m}(\tilde{X})}\left[\sigma_{k_{1}}-\sigma\right]\left(\tilde{X}_{s-}\right) d s\right|>\frac{\varepsilon}{3}\right]+\tilde{\mathbf{P}}\left[\tau_{m}\left(\tilde{X}^{n}\right)<t\right]+\tilde{\mathbf{P}}\left[\tau_{m}(\tilde{X})<t\right] .
\end{gathered}
$$


The first term on the right side of the inequality above converges to 0 as $n \rightarrow \infty$ by Chebyshev's inequality and the Lebesgue bounded convergence theorem. To show the convergence to 0 as $n \rightarrow \infty$ of the second and third terms we first use Chebyshev's inequality and then Corollary 3.5 and Lemma 4.2, respectively. We obtain

$$
\tilde{\mathbf{P}}\left[\left|\int_{0}^{t \wedge \tau_{m}\left(\tilde{X}^{n}\right)}\left[\sigma_{k_{1}}-\sigma_{n}\right]\left(\tilde{X}_{s-}^{n}\right) d s\right|>\frac{\varepsilon}{3}\right] \leq \frac{3}{\varepsilon} N\left\|\sigma_{k_{1}}-\sigma_{n}\right\|_{2, m}
$$

and

$$
\tilde{\mathbf{P}}\left[\left|\int_{0}^{t \wedge \tau_{m}(\tilde{X})}\left[\sigma_{k_{1}}-\sigma\right]\left(\tilde{X}_{s-}\right) d s\right|>\frac{\varepsilon}{3}\right] \leq \frac{3}{\varepsilon} N\left\|\sigma_{k_{1}}-\sigma\right\|_{2, m}
$$

where the constant $N$ depends on $\Lambda, m, t$, and $\alpha$ only. It follows from the definition of the sequence $\sigma_{n}$ that, for any $m \in \mathbb{N}, \sigma_{n} \rightarrow \sigma$ as $n \rightarrow \infty$ in the $L_{2, m}$-norm. Then, passing to the limit in (4.8) and (4.9) first $n \rightarrow \infty$ and then $k_{1} \rightarrow \infty$, we obtain that the right sides of (4.8) and (4.9) converge to 0 .

Because of the property (4.7),

$$
\tilde{\mathbf{P}}\left[\tau_{m}\left(\tilde{X}^{n}\right)<t\right] \rightarrow \tilde{\mathbf{P}}\left[\tau_{m}(\tilde{X})<t\right] \quad \text { as } \quad n \rightarrow \infty .
$$

Therefore, the last two terms can be made arbitrarily small by choosing large enough $m$ for all $n$ due to the fact that the sequence of processes $\tilde{X}^{n}$ satisfies the property (4.3). This verifies (4.6). Thus, we have proven the existence of the process $\tilde{X}$ that solves the equation (4.1).

Corollary 4.3. Suppose that the coefficient a is bounded. Then, for any initial value $x_{0} \in \mathbb{R}$, there exists a solution of the equation (1.4).

\section{ACKNOWLEDGEMENT}

The author would like to express his gratitude to Professor Henrikas Pragarauskas for the valuable suggestions and comments on the subject of Krylov's estimates for stable processes. I also thank the referee for the helpful remarks.

\section{REFERENCES}

[A] D. Aldous, Stopping times and tightness, Ann. Prob., 6 (1978), 335-340. MR0474446 (57:14086)

[AP] S. Anulova and H. Pragarauskas, On strong Markov weak solutions of stochastic equations, Liet. Math. Rinkinys, XVII (1977), 5-26. MR58:31397

[B] R. Bass, Stochastic differential equations driven by symmetric stable processes, Séminaire de Probabilités, XXXVI, Lecture Notes in Math., 1801, 302-313, Springer, Berlin, 2003. MR1971592 (2004b:60143)

[DM] C. Dellacherie and P.A. Meyer, Probabilities et Potentiels B, Hermann, Paris, 1980. MR566768 (82b:60001)

[ES1] H.J. Engelbert and W. Schmidt, On one-dimensional stochastic differential equations with generalized drift, Lect. Notes Control Inf. Sci., 69, 143-155, Springer, Berlin, 1985. MR798317 (86m:60144)

[ES2] _ Strong Markov continuous local martingales and solutions of one-dimensional stochastic differential equations III, Math. Nachr., 151 (1991), 149-197. MR1121203 (92m:60044)

[IW] N. Ikeda and S. Watanabe, Stochastic Differential Equations and Diffusion Processes, North-Holland Publ., Tokyo, 1989. MR1011252 (90m:60069)

[J] J. Jacod, Calcul Stochastique et Problèmes de Martingales. Lect. Notes Math., Vol. 714, Springer, Berlin, 1979. MR542115 (81e:60053) 
[K] O. Kallenberg, Foundations of Modern Probability, Springer, Berlin, 1997. MR1464694 (99e:60001)

[KR] N.V. Krylov, Controlled Diffusion Processes, Springer, New York, 1980. MR601776 (82a:60062)

[LM] J.P. Lepeltier and B. Marchal, Probléme des martingales et équations différentielles stochastiques associées á un opérateur intégro-différentiel, Annales IHP, Vol. 12, No. 1 (1976), 43-103. MR0413288 (54:1403)

[M] A.V. Melnikov, Stochastic equations and Krylov's estimates for semimartingales, Stochastics and Stoch. Rep., 10 (1983), 81-102. MR716817 (85j:60113)

[PO] N.I. Portenko, Some perturbations of drift-type for symmetric stable processes, Random Oper. and Stoch. Equ., Vol. 2, No. 3 (1994), 211-224. MR1310558 (95k:60144)

[PR] H. Pragarauskas, On $L^{p}$-estimates of stochastic integrals, In: "Probab. Theory and Math. Statist.", B.Grigelionis et al. (eds.), 579-588, VSP, Utrecht/TEV, Vilnius, 1999.

[PZ] H. Pragarauskas and P.A. Zanzotto, On one-dimensional stochastic differential equations driven by stable processes, Liet. Mat. Rink., 40 (2000), 1-24. MR2003a:60091

[RY] D. Revuz and M. Yor, Continuous Martingales and Brownian Motion, Springer, Berlin, 1999. MR1725357 (2000h:60050)

[RW] J. Rosiński and W. Woyczyński, On Itô stochastic integration with respect to p-stable motion: inner clock, integrability of sample paths, double and multiple integrals, Ann. Probab., 14 (1986), 271-286. MR815970 (87h:60109)

[TTW] H. Tanaka, M. Tsuchiya, S. Watanabe, Perturbation of drift-type for Levy processes, Journal Math. Kyoto University, 14, No. 1 (1974), 73-92. MR0368146 (51:4388)

[Z1] P.A. Zanzotto, Representation of a class of semimartingales as stable integrals, Theory Probab. Appl., Vol. 43, No. 4 (1998), 808-818. MR1692452 (2000a:60107)

[Z2] P.A. Zanzotto, On stochastic differential equations driven by Cauchy process and the other stable Lévy motions, Ann. Probab., Vol. 30, No. 2 (2002), 802-825. MR1905857 (2003d:60120)

Department of Natural and Applied Sciences, University of Wisconsin-Green Bay, 2420 Nicolet Drive, Green Bay, Wisconsin 54311-7001

E-mail address: kurenokv@uwgb.edu 